\title{
Differential DNases are selectively used in neuronal apoptosis depending on the differentiation state
}

\author{
D Shiokawa ${ }^{1}$ and S Tanuma ${ }^{\star, 1,2}$ \\ 1 Department of Biochemistry, Faculty of Pharmaceutical Sciences, Tokyo \\ University of Science, 2641 Yamazaki, Noda, Chiba 278-8510, Japan \\ 2 Genome and Drug Research Center, Tokyo University of Science, 2669 \\ Yamazaki, Noda, Chiba 278-0022, Japan \\ * Corresponding author: S Tanuma, Department of Biochemistry, Faculty of \\ Pharmaceutical Sciences, Tokyo University of Science, 2641 Yamazaki, Noda, \\ Chiba 278-8510, Japan. Tel: + 81-4-7124-1501; Fax: + 81-4-7121-3620; \\ E-mail: tanuma@rs.noda.tus.ac.jp
}

Received 22.1.04; accepted 04.3.04; published online 28.5.04 Edited by $\mathrm{H}$ Ichijo

\begin{abstract}
In this study, we investigate the roles of two apoptotic endonucleases, CAD and DNase $\gamma$, in neuronal apoptosis. High expression of CAD, but not DNase $\gamma$, is detected in proliferating N1E-115 neuroblastoma cells, and apoptotic DNA fragmentation induced by staurosporine under proliferating conditions is abolished by the expression of a caspaseresistant form of ICAD. After the induction of neuronal differentiation, CAD disappearance and the induction of DNase $\gamma$ occur simultaneously in N1E-115 cells. Apoptotic DNA fragmentation that occurs under differentiating conditions is suppressed by the downregulation of DNase $\gamma$ caused by its antisense RNA. The induction of DNase $\gamma$ is also observed during neuronal differentiation of PC12 cells, and apoptotic DNA fragmentation induced by NGF deprivation is inhibited by the antisense-mediated downregulation of DNase $\gamma$. These observations suggest that DNA fragmentation in neuronal apoptosis is catalyzed by either CAD or DNase $\gamma$ depending on the differentiation state. Furthermore, DNase $\gamma$ is suggested to be involved in naturally occurring apoptosis in developing nervous systems.

Cell Death and Differentiation (2004) 11, 1112-1120.

doi:10.1038/sj.cdd. 4401454

Published online 28 May 2004
\end{abstract}

Keywords: apoptosis; differentiation; DNA fragmentation; DNase, neuron

\footnotetext{
Abbreviations: CAD, caspase-activated DNase; CBB, coomassie brilliant blue; CNS, central nervous system; DFF, DNA fragmentation factor; DIC, differential interference contrast; DM, differentiation medium; DPBS, Dulbecco's modified phosphatebuffered saline; FITC, fluorescein isothiocyanate; GM, growth medium; ICAD, inhibitor of CAD; mAb, monoclonal antibody; NF, neurofilament; PNS, peripheral nervous system; STS, staurosporine
}

\section{Introduction}

During the development of the nervous system in vertebrates, early neurons are produced in excess by the differentiation of their progenitor neuroblasts. The early neurons can survive and extend axons independently of neurotorophic factors; however, when the axons make contact with their targets, they become dependent on target-derived neurotorophic factors for survival. ${ }^{1}$ At this time, a substantial number of innervating neurons die by apoptosis because of the limited supply of neurotrophic factors in the target fields. ${ }^{1-3}$ The physiological loss of developing neurons, known as naturally occurring neuronal apoptosis, is observed both in the central nervous system (CNS) and the peripheral nervous system (PNS), and is an important process for matching the number of neurons to the requirements of their targets. ${ }^{1-3}$

Apoptosis is a cellular suicidal program by which potentially harmful or no longer needed cells are individually eliminated to maintain healthy homeostasis in multicellular organisms. ${ }^{4-6}$ Apoptosis is distinguished from necrosis by its specific morphologies, such as loss of cell volume and chromatin condensation, and biochemical markers, including the externalization of membrane phosphatidylserine, the cleavage of death substrates by activated caspases, and the degradation of genomic DNA into nucleosomal fragments. ${ }^{7-9}$ The occurrence of nucleosomal DNA fragmentation has been recognized as an important feature of apoptosis, and the ladder configuration visualized by agarose gel electrophoresis is frequently used as reliable evidence of apoptosis. ${ }^{10}$

To date, several mammalian DNases have been suggested to be involved in the process of apoptosis. CAD/DFF40, DNase I, DNase $\gamma /$ DNAS1L3, and endonuclease G are suggested to produce oligonucleosomal DNA fragmentation in dying cells. ${ }^{11-16}$ Cells undergoing apoptosis are rapidly eliminated by phagocytosis in vivo, and the DNA of engulfed cells is further digested by a lysosomal acid endonuclease, DNase $11 .{ }^{17}$ Among the candidates for nucleosomal DNA fragmentation, the role of CAD/DFF40 has been well documented in many apoptotic cases both in vivo and in vitro. ${ }^{18-20}$ However, it remains controversial whether the apoptotic DNase is always the same or differs depending on cell types, differentiation state, and/or apoptotic stimuli.

DNA fragmentation factor (DFF) is a heterodimeric complex of DFF40 and DFF45 that was purified from the $S 100$ fraction of HeLa cells on the basis of its activity to induce DNA ladder formation in a cell-free apoptosis system. ${ }^{13}$ Murine homologues of DFF40 and DFF45, caspase-activated DNase (CAD) and inhibitor of CAD (ICAD), respectively, have been isolated and characterized. ${ }^{11}$ CAD/DFF40 exists in living cells as an inactive complex with its natural inhibitor ICAD/DFF45. Upon the induction of apoptosis, CAD/DFF40 is released from the complex by caspase-3-mediated cleavage of ICAD/ DFF45, and catalyzes nucleosomal DNA fragmentation in dying cells. ${ }^{11}$ 
On the other hand, a nuclear $\mathrm{Ca}^{2+} / \mathrm{Mg}^{2+}$-dependent DNase has long been suggested as an apoptotic DNase, ${ }^{21,22}$ and we identified and purified a novel $33 \mathrm{kDa}$ endonuclease DNase $\gamma^{23}$ Sequence analysis of its cDNA revealed that DNase $\gamma$ is a member of the mammalian DNase I family of DNases. ${ }^{16}$ DNase $\gamma$ is unique among family members in its ability to produce DNA fragmentation during apoptosis, ${ }^{24,25}$ however, the apoptotic situations in which DNase $\gamma$ catalyzes DNA ladder formation remain largely unknown. In a recent study, it was demonstrated that the induction of DNase $\gamma$ takes place in $\mathrm{C} 2 \mathrm{C} 12$ cells during myogenic differentiation, and the suppression of DNase $\gamma$ by the stable expression of its antisense RNA results in the absence of DNA ladder formation in apoptosis that occurs spontaneously during differentiation. ${ }^{26}$ Thus, DNase $\gamma$ has been shown to be indispensable for DNA fragmentation in the differentiationinduced apoptosis of $\mathrm{C} 2 \mathrm{C} 12$ myoblasts.

In this study, we examined the roles of CAD and DNase $\gamma$ in neuronal apoptosis using the differentiation system of N1E115 murine neuroblastoma cells. We found that nucleosomal DNA fragmentation is catalyzed by CAD and DNase $\gamma$ under proliferating and differentiating conditions, respectively. Furthermore, the role of DNase $\gamma$ in NGF deprivation-induced apoptosis is demonstrated using neuronally differentiated PC12 cells. This is the first report describing the selective usage of distinct apoptotic DNases depending on the degree of differentiation. Moreover, our results suggest that DNase $\gamma$ is involved in naturally occurring apoptosis in developing nervous system.

\section{Results}

\section{Characterization of cell death occurring spontaneously during the neuronal differentiation of N1E-115 cells}

Figure 1 shows the neuronal differentiation of N1E-115 cells induced by serum withdrawal. Proliferating N1E-115 cells exhibit a fibroblast-like morphology with a faint expression of neurofilament heavy chain (NF-H) in cytoplasm (Figure 1a). Neurite outgrowth and upregulation of NF-H were initiated one day after the induction of neuronal differentiation (Figure 1b), and most of the cells were began to show an NF-H-positive neuronal morphology on day 4 (Figure 1C). At the same time, a substantial number of dead cells appeared in the differentiating culture (Figure 1c, white arrows). The dead cells detached easily from the coverslips or culture dishes, so that only a small number of dead cells could be detected by fluorescence microscopy.

The occurrence of cell death is clearly demonstrated by the trypan blue staining of the whole culture (Figure $1 \mathrm{e}-\mathrm{h}$ ). There were few floating dead cells at day 1 (Figure 1f), however, the degree of cell death increased with time and was observed most frequently at day 4 (Figure $1 \mathrm{~g}$ ). The appearance of newly dead cells declined after this period, and scarcely detected at day 10 (Figure $1 \mathrm{~h}$ ). At this time, most living cells had become terminally differentiated neurons with a rounded cell body, constricted nuclear morphology, and a long unipolar neurite (Figure 1d). The expression of NF-H was detected at high levels within neurites, but scarcely in cell bodies (Figure 1d). In

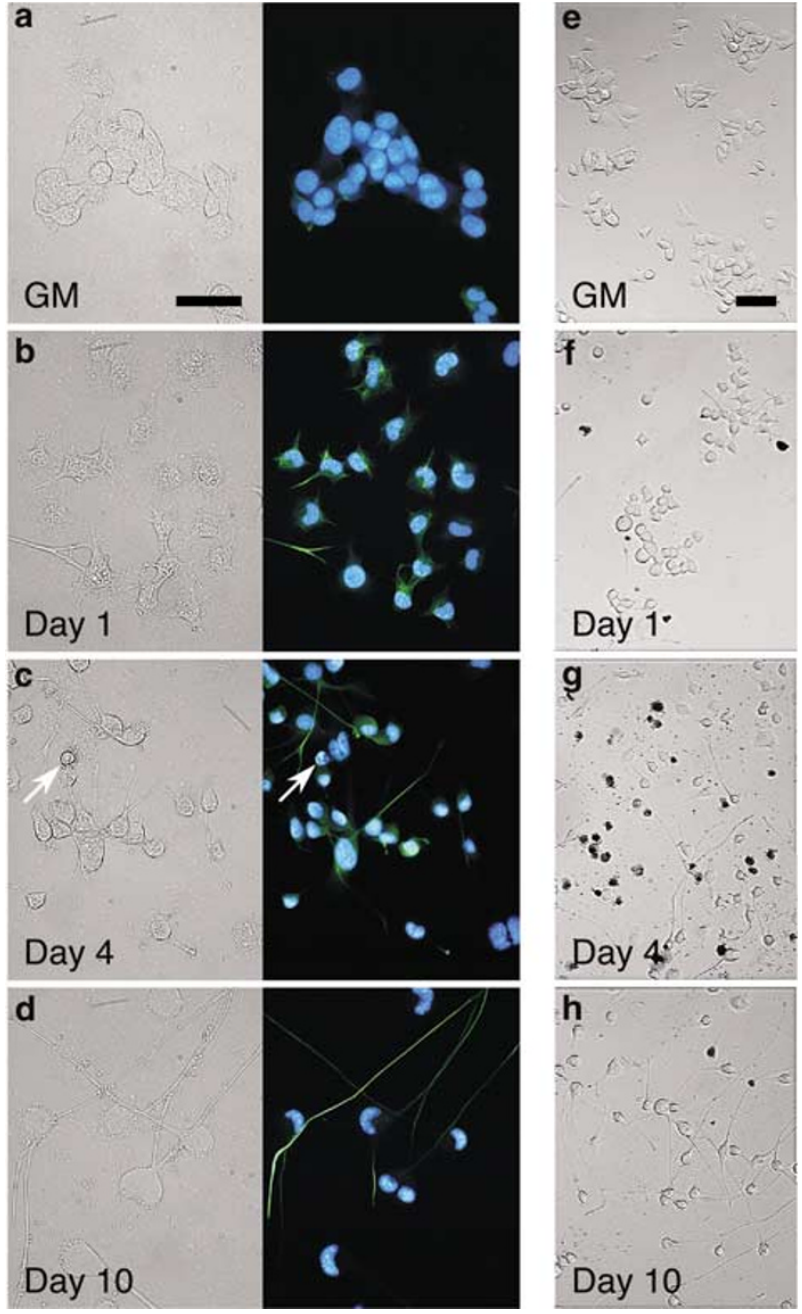

Figure 1 Serum withdrawal induces both neuronal differentiation and cell death in N1E-115 cells. Cultures maintained in growth medium (GM) were shifted to differentiation medium (DM) and cultured for the indicated number of days. (ad) The resulting cells were successively stained with antineurofilament-H (NF-H) antibody and FITC-conjugated secondary antibody as described under Materials and Methods. Nuclei were counterstained with Hoechst dye. Differential interference contrast (DIC) microscopy revealed their morphological changes (left half). NF-H (green) and nuclei (blue) were observed by fluorescence microscopy and their overlayed images are shown on the right half. (e-h) Trypan blue was added to cultures maintained in GM or DM for the indicated number of days, and the cells were observed under DIC microscopy as described in Materials and Methods. Dead cells are selectively stained by the dye. The black bars represent $50 \mu \mathrm{m}$

this experimental system, $70-80 \%$ of the initial cells were lost to cell death during the differentiation process (Figure 2a).

To determine the form of cell death associated with neuronal differentiation, floating dead cells were collected from the culture and subjected to further characterization. The spontaneous death of differentiating N1E-115 cells was found to satisfy many important criteria of apoptosis: cleavage of poly(ADP-ribose) polymerase-1 (PARP-1) into an $85 \mathrm{kDa}$ fragment was detected by Western blot (Figure 2b), nuclear condensation and externalization of membrane phosphatidylserine were evidenced by Hoechst and fluorescein isothiocyanate (FITC)-annexin V staining, respectively (Figure 2c), and an agarose gel analysis revealed the appearance of 


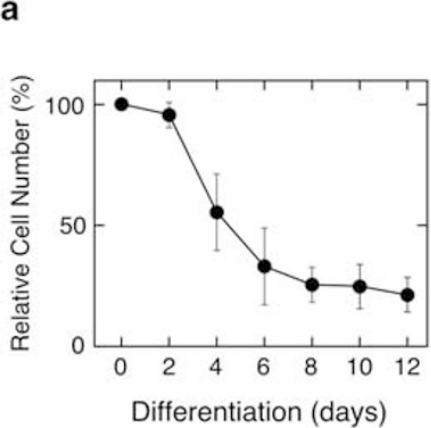

C
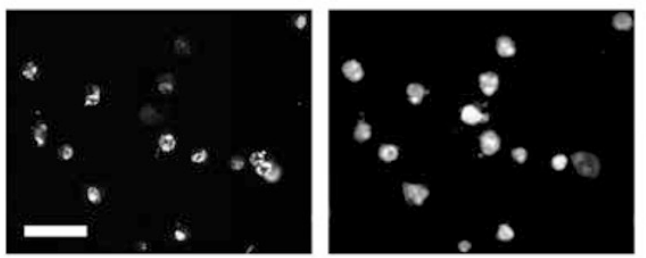

d

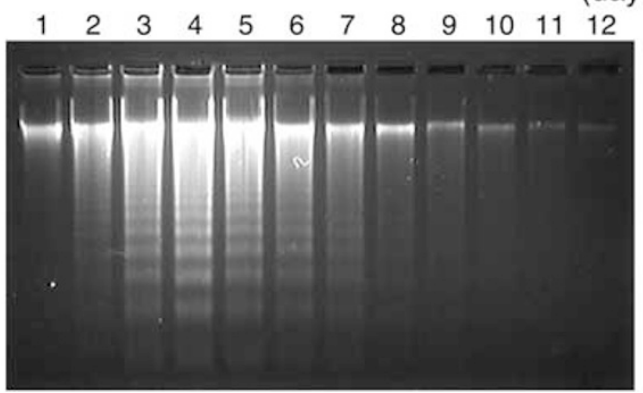

Figure 2 Characterization of naturally occurring cell death associated with neuronal differentiation of N1E-115 cells. (a) Proliferating cultures were shifted to DM and maintained for 12 days. Living cells on the indicated day were counted and the results are given relative to the initial cell number at day 0 . Values are shown as mean \pm S.D. $(n=3)$. (b) Living cells maintained in GM (lane 1$)$ and detached cells collected from the differentiation culture on day 4 (lane 2) were subjected to Western blot analysis for PARP-1 as described under Materials and Methods. Closed and open arrowheads indicate intact $(116 \mathrm{kDa})$ and cleaved $(85 \mathrm{kDa}$ ) forms of PARP-1, respectively. (c) Detached cells on differentiation day 4 were collected, stained with FITC-annexin V and Hoechst dye, and observed by fluorescence microscopy as described under Materials and Methods. Nuclear condensation and externalization of phosphatidylserine were detected by Hoechst (left panel) and FITC-annexin V (right panel) staining, respectively. The white bar represents $50 \mu \mathrm{m}$. (d) Proliferating cells were shifted to DM and cultured for 12 days with daily medium replacement. Detached cells were recovered from the medium on the indicated day, and the same aliquots of genomic DNA prepared from cells were analyzed by $1.8 \%$ agarose gel electrophoresis as described under Materials and Methods

nucleosomal DNA fragmentation, a sign of apoptotic DNase activation (Figure $2 \mathrm{~d}$ ). The same results were obtained for staurosporine (STS)-induced cell death under both proliferating and differentiating conditions (data not shown). On the basis of these results, the death of N1E-115 cells, both that occurring spontaneously during differentiation and that induced by STS-treatment, is judged to be typical apoptosis.

\section{Distinct transcriptional controls of CAD and DNase $\gamma$ during neuronal differentiation of N1E-115 cells}

To identify the DNase(s) responsible for apoptotic DNA fragmentation in N1E-115 cells, we analyzed the expression of two apoptotic DNases, CAD and DNase $\gamma$, during neuronal differentiation (Figure 3a). RT-PCR analyses detected the mRNAs for both CAD and its inhibitor, ICAD, in N1E-115 cells under proliferating conditions. Although the expression of ICAD remained unchanged during differentiation, the mRNA for CAD decreased in the early phase of differentiation and was scarcely detected in differentiated neurons. In contrast, the expression of the DNase $\gamma$ mRNA was quite low in proliferating cells; however, it began to increase shortly after the induction of differentiation, and was detected at high levels in neuronally differentiated cells. The downregulation of CAD and the induction of DNase $\gamma$ were also confirmed by Western blot and activity gel assay, respectively (Figure $3 b$ ). The band intensities representing CAD and DNase $\gamma$ proteins appeared to correlate well with each mRNA expression levels. These results indicate that the primary apoptotic DNase in proliferating N1E-115 cells is CAD, and it is replaced by DNase $\gamma$ during neuronal differentiation. That is, our findings suggest that apoptotic DNA fragmentation in N1E-115 cells is produced by distinct DNases depending on the degree of differentiation.

a

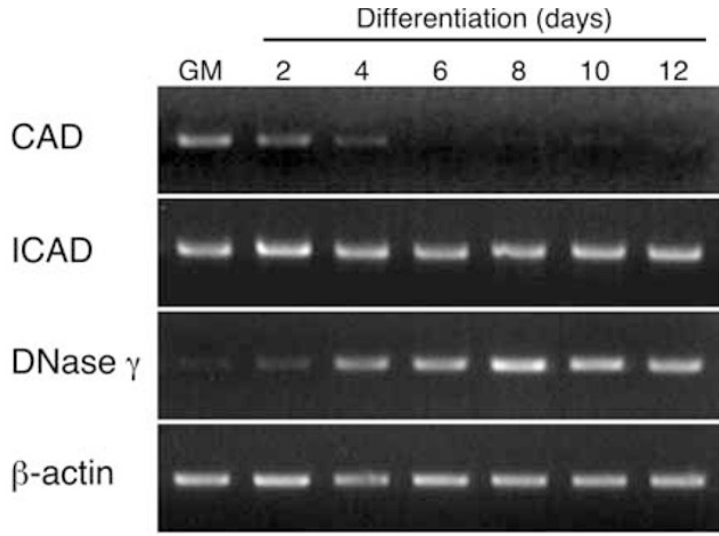

b

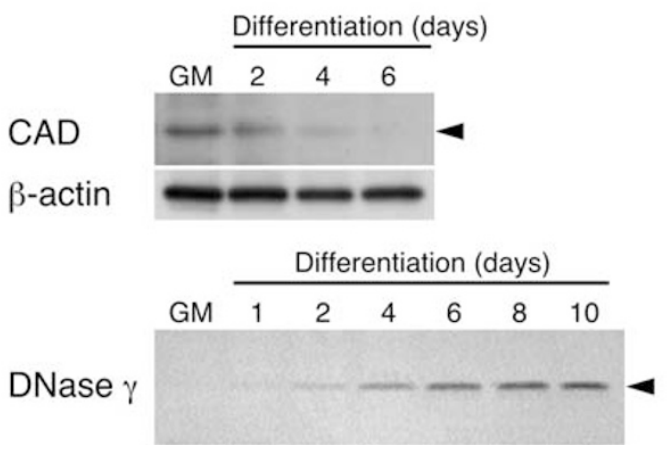

Figure 3 Changes in apoptotic DNase expression during neuronal differentiation of N1E-115 cells. (a) RT-PCR analyses for the indicated mRNAs were performed on proliferating (GM) and differentiating (differentiation days 2-12) N1E-115 cells as described under Materials and Methods. cDNAs generated from the cells were normalized for $\beta$-actin expression. (b) N1E-115 cells grown in GM or cultured in DM for indicated days were subjected to Western analysis for CAD (upper panels) and activity gel assay for DNase $\gamma$ (lower panel) as described under Materials and Methods. The closed arrowhead indicates the bands representing CAD protein and DNase $\gamma$ activity, respectively 


\section{Involvement of CAD in STS-induced apoptosis in proliferating N1E-115 cells}

As an initial approach to evaluate this hypothesis, we examined the role of CAD in proliferating N1E-115 cells. $\mathrm{CAD}$ is activated by the caspase-3-mediated cleavage of ICAD at two aspartic acid residues, Asp117 and 224. ${ }^{11}$ Therefore, the apoptotic DNA fragmentation produced by CAD is selectively inhibited when ICAD is doubly mutated at those caspase-cleavage sites. ${ }^{19}$

STS-treatment induced proliferating $\mathrm{N} 1 \mathrm{E}-115$ cells to undergo apoptosis. Nucleosomal DNA fragmentation that appeared in control cells correlated well with the disappearance of intact ICAD (Figure $4 a$ and $b$, left panels), whereas no DNA laddering could be seen in cells stably transfected with ICAD-CR, a caspase-resistant form of ICAD (Figure $4 a$, right panel). Western blot analysis confirmed that ICAD-CR, but not endogenous ICAD, remained intact throughout the time course (Figure $4 \mathrm{~b}$, right panel). In both control and ICAD-CR cells, the kinetics of PARP-1 cleavage was found to be almost identical (Figure 4c). Thus, the overexpression of ICAD-CR is shown to suppress DNA fragmentation without affecting the induction of apoptosis in proliferating N1E-115 cells. The same results were obtained with another inducers of apoptosis, actinomycin D and cycloheximide (data not shown).

On the basis of these results, apoptotic DNA fragmentation in proliferating N1E-115 cells is shown to be produced by CAD.

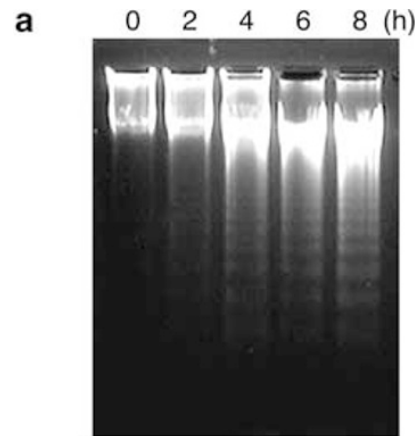

Puro

b

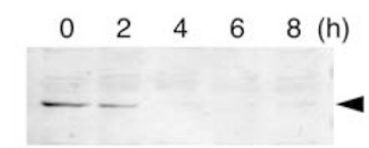

C

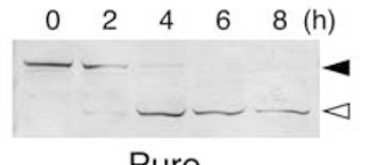

Puro

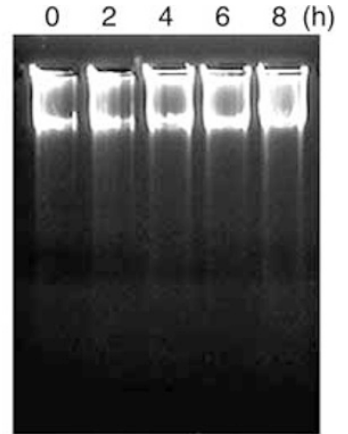

ICAD-CR

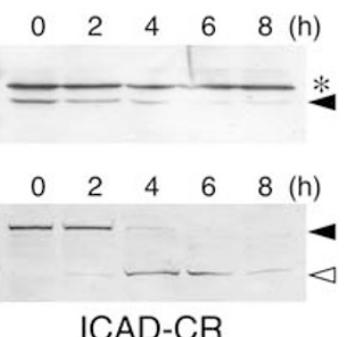

ICAD-CR
Figure 4 Expression of ICAD-CR inhibits STS-induced apoptotic DNA fragmentation in proliferating N1E-115 cells. N1E-115 mock-transfectants (Puro) or stably transfected with caspase-resistant ICAD (ICAD-CR) were treated with $0.5 \mu \mathrm{M}$ STS for $0-8 \mathrm{~h}$ under proliferating conditions. The resulting cells were harvested at the indicated times and subjected to DNA fragmentation analysis (a) and Western blot (b, c) as described under Materials and Methods. Western analyses were performed using anti-ICAD pAb (b) or anti-PARP pAb (c). Closed and open arrowheads indicate intact and cleaved antigens, respectively. The asterisk shows the bands representing the C-terminally Myc and His-tagged ICAD-CR protein

\section{DNase $\gamma$ catalyzes DNA fragmentation in apoptosis associated with neuronal differentiation of N1E- 115 cells}

The involvement of DNase $\gamma$ in apoptosis under differentiating conditions was examined using DNase $\gamma$-knockdown cells established by the stable expression of its antisense RNA. Activity gel analysis shows the suppression of DNase $\gamma$ activity in the antisense cells after 4 days of differentiation (Figure 5a). DNase $\gamma$ activity in mock transfectants was detected normally at the same level as in parental cells. In contrast, DNase $\gamma$ activity in the antisense cells was effectively suppressed as compared with control cells (Figure 5a). This is not due to artificial defects in the ability to differentiate because neurite outgrowth was observed normally in both control and antisense cells (Figure 5b). The suppression of DNase $\gamma$ was also shown by indirect immunofluorescence. Positive staining for DNase $\gamma$ was observed in the apoptotic nuclei of control cells, but not in the DNase $\gamma$-antisense cells (Figure 5b, white arrows). We used a monoclonal antibody (mAb) that recognizes activated DNase $\gamma$ located in apoptotic nuclei. ${ }^{27,26}$ Therefore, the negative staining of living cells does not mean the absence of DNase $\gamma$ in control cells.

By using the antisense cells, we evaluated the role of DNase $\gamma$ in the differentiation-induced apoptosis of N1E-115 cells. The downregulation of DNase $\gamma$ had no apparent effect on the induction of spontaneous apoptosis during differentiation (Figure 5c); however, apoptotic ladder formation was strongly suppressed in the DNase $\gamma$-antisense cells as compared with control (Puro) or ICAD-CR cells (Figure 5d). The same results were also obtained in another DNase $\gamma$ antisense clones. As shown in Figure 5e, the magnitudes of DNA ladder formation (upper panels) were found to correlate well with DNase $\gamma$ activities detected in the cells (lower panels).

\section{DNase $\gamma$ in neuronally differentiated N1E-115 cells is still able to produce apoptotic DNA fragmentation}

The above observations provide direct evidence for the involvement of DNase $\gamma$ in spontaneous apoptosis in early differentiating neurons. However, the expression of DNase $\gamma$ is still high in the late phase of differentiation when spontaneous apoptosis is scarcely observed. This strongly suggests that DNase $\gamma$ also plays an important role in apoptosis of terminally differentiated neurons. Thus, we examined whether DNase $\gamma$ is still functional by able to produce apoptotic ladders in such cells. N1E-115 cells on differentiation day 8 were found to be relatively insensitive to the toxic effects of STS as compared with cells grown in proliferating culture (Figure 6a); however, apoptosis in neuronally differentiated N1E-115 cells was still accompanied by DNA ladder formation (Figure 6b, left panel). Although DNase $\gamma$-antisense cells underwent apoptosis with the same kinetics as control cells (Figure 6a), DNA fragmentation was scarcely observed during STS-induced apoptosis (Figure 6b, right panel). On the basis of these results, DNase $\gamma$ expressed in differentiated N1E-115 cells is shown still to be capable of 
catalyzing DNA ladder formation when the cells are forced to undergo apoptosis.

\section{DNase $\gamma$ is induced during the neuronal differentiation of PC12 cells and catalyzes apoptotic DNA fragmentation induced by NGF withdrawal}

The above finding tempted us to examine the role of DNase $\gamma$ in apoptosis induced by neurotrophic factor deprivation, because this is one of the most important forms of physiological apoptosis in differentiated neurons. Unfortunately, N1E-115 cells survive in differentiation culture without any external supply of neurotrophic factors. Therefore, we a

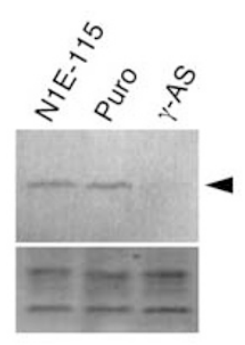

b

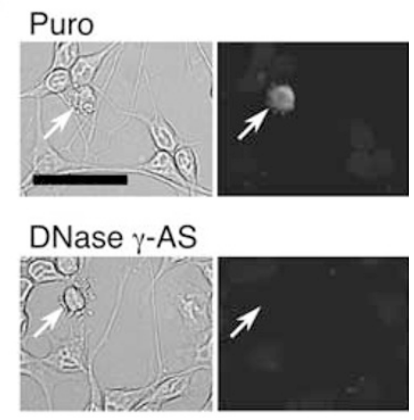

C

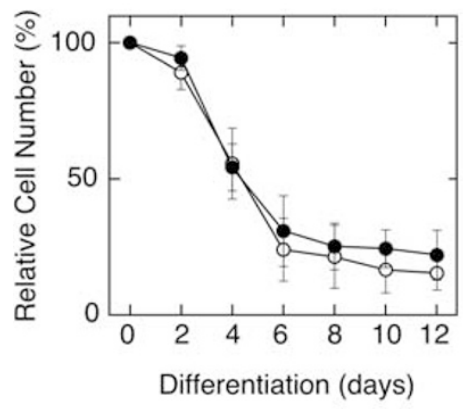

d

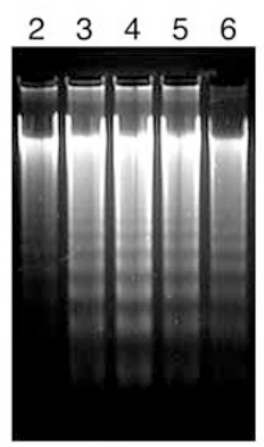

Puro e

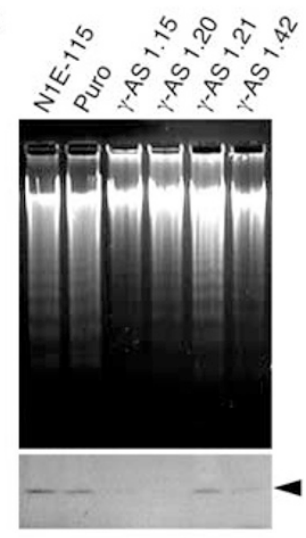

(days)

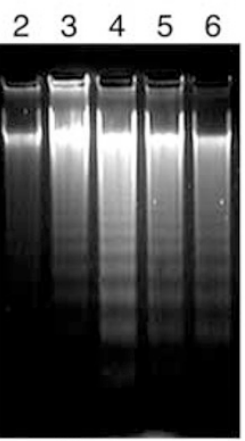

ICAD-CR

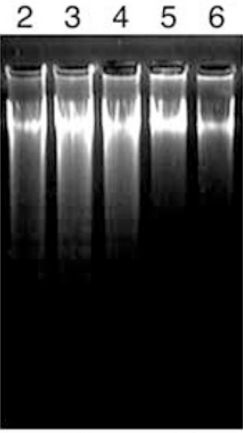

DNase $\gamma$-AS used a rat pheochromocytoma cell line, PC12 cells, for this purpose.

NGF-treatment induced neuronal differentiation in $\mathrm{PC} 12$ cells. RT-PCR analysis revealed this differentiation to be accompanied by the induction of DNase $\gamma$ (Figure 7a). The activation of DNase $\gamma$ expression was preceded by the initiation of neurite outgrowth, and, at day 6, DNase $\gamma$ activity in PC12 cells became detectable by activity gel assay at the same level as detected in PC12/Puro cells (data not shown).

To define the role of DNase $\gamma$ in neuronally differentiated PC12 cells, we established DNase $\gamma$-knockdown cells and analyzed their phenotype in apoptosis induced by NGF deprivation. The suppression of DNase $\gamma$ by the stable expression of its antisense RNA is shown by the activity gel assay (Figure $7 \mathrm{~b}$ ). The band representing DNase $\gamma$ activity was detected in control cells, but not in the antisense cells under differentiating conditions.

Both control and the antisense PC12 cells normally undergo neuronal differentiation when the cultures are shifted to DM supplemented with NGF (Figure 7c). After the induction of differentiation, the cells start to extend multiple neurites, and at day 6 , they are terminally differentiated and become dependent on NGF for survival. NGF deprivation induced apoptosis in these neuronal PC12 cells; shrunken dead cells appeared in the cultures after $24 \mathrm{~h}$ (Figure 7c), and most cells became apoptotic at $48 \mathrm{~h}$ post-NGF deprivation. As shown in Figure $7 d$, the apoptosis of neuronally differentiated control cells was accompanied by nucleosomal DNA fragmentation, whereas such ladder formation was scarcely detected in antisense cells. We observed no apparent difference in the magnitude of cell death between control and antisense cells (Figure 7c, right panels), and this is supported by the same kinetics of PARP cleavage (Figure 7d, lower panels). On the basis of these results, DNase $\gamma$ is shown to be indispensable for apoptotic DNA fragmentation during NGF deprivationinduced apoptosis in neuronally differentiated PC12 cells.

Figure 5 Suppression of DNA fragmentation during differentiation-induced apoptosis by the antisense RNA-mediated downregulation of DNase $\gamma$. (a) Parental cells (N1E-115), mock transfectants (Puro), and DNase $\gamma$-antisense clone $1.15(\gamma$-AS) were cultured in DM for 4 days, and their DNase $\gamma$ activities were assayed by the activity gel method as described under Materials and Methods. For quantitation of lane loading, the bands representing core histons on the CBB stained gel are shown at the bottom for comparison. The closed arrowhead indicates the band representing DNase $\gamma$ activity. (b) N1E-115 mocktransfectants (Puro) and DNase $\gamma$-antisense clone 1.15 (DNase $\gamma$-AS) were cultured in DM for 4 days, stained successively with anti-DNase $\gamma$ mAb and FITCconjugated secondary antibody, and observed by fluorescence microscopy as described under Materials and Methods. DIC microscopy revealed the morphologies (left panels). The fluorescence of FITC indicates the nuclear localization of DNase $\gamma$ in apoptotic nuclei (right panels, white arrows). The black bar represents $50 \mu \mathrm{m}$. (c) N1E-115 mock-transfectants (closed symbols) and DNase $\gamma$-antisense clone 1.15 (open symbols) were cultured in DM for 12 days. Living cells on the indicated days were counted and the results are given relative to the initial cell number at day 0 . Values are shown as mean \pm S.D. $(n=3)$. (d) N1E-115 mock-transfectants (Puro), ICAD-CR cells (ICAD-CR), and DNase $\gamma$ antisense clone 1.15 (DNase $\gamma$-AS) were cultured in DM with daily medium replacement, and detached cells collected on the indicated days were subjected to DNA fragmentation analysis as described under Materials and Methods. (e) Parental cells (N1E-115), mock transfectants (Puro), and the indicated DNase $\gamma$ antisense clones $(\gamma-\mathrm{AS})$ were cultured in $\mathrm{DM}$ with daily medium replacement. Detached and attached cells were collected at day 4, and subjected to DNA fragmentation analysis (upper panel) and activity gel assay (lower panel), respectively, as described under Materials and Methods 
b

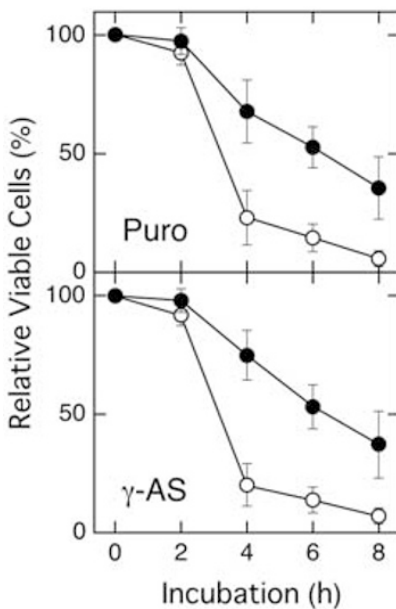

$0 \quad 4 \quad 8$

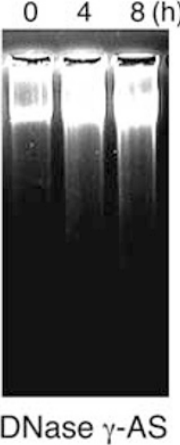

Figure 6 Suppression of STS-induced apoptotic DNA fragmentation in differentiated DNase $\gamma$-antisense cells. (a) N1E-115 mock-tarnsfectants (Puro) and DNase $\gamma$-antisense cells ( $\gamma$-AS), either maintained in GM (open symbols) or allowed to differentiate for 8 days (closed symbols), were treated with $0.5 \mu \mathrm{M}$ STS for $8 \mathrm{~h}$. Living cells at the indicated times were counted and the results are shown relative to the initial cell number at $0 \mathrm{~h}$. Values are shown as mean \pm S.D. $(n=3)$. (b) N1E-115 mock-transfectants (Puro) and DNase $\gamma$-antisense cells (DNase $\gamma$-AS) were allowed to differentiate for 8 days, and treated with $0.5 \mu \mathrm{M}$ STS for $8 \mathrm{~h}$. Total cells harvested at the indicated times were subjected to DNA fragmentation analysis as described under Materials and Methods

\section{Discussion}

Apoptosis in postmitotic neurons and its regulation by neurotrophic factors has been well examined both in vivo and in vitro; however, only a few studies have focused on spontaneous apoptosis associated with the process of neurogenesis or neuronal differentiation. This is considered to be due to the difficulty of detecting such apoptosis in vivo, and the lack of appropriate experimental systems in vitro. A mouse neuroblastoma cell line, N1E-115, is one of the most

Figure 7 Involvement of DNase $\gamma$ in NGF-deprivation-induced apoptosis in neuronally differentiated PC12 cells. (a) RT-PCR analysis for DNase $\gamma$ mRNA was performed on proliferating (GM) and differentiating (differentiation days 1-6) PC12 cells as described under Materials and Methods. cDNAs generated from the indicated cells were normalized for $\beta$-actin expression. (b) DNase $\gamma$ activities in PC12 cells stably expressing the antisense RNA for DNase $\gamma$. Mock transfectants (Puro) and DNase $\gamma$-antisense cells $(\gamma$-AS), maintained in GM (GM) or cultured in DM for 6 days (DM), were subjected to the activity gel assay and their DNase $\gamma$ activities were detected as described under Materials and Methods. For quantitation of lane loading, the bands representing core histons on the CBB stained gel are shown at the bottom for comparison. The closed arrowhead indicates the band representing DNase $\gamma$ activity. (c) PC12 mocktransfectants (upper panels) and DNase $\gamma$-antisense cells (lower panels) were observed by phase-contrast microscopy: left panels, proliferating cells; center panels, 6 days differentiated cells; right panels, cells differentiated for 6 days and further cultured in the absence of NGF for $24 \mathrm{~h}$. The black bar represents $100 \mu \mathrm{m}$. (d) PC12 mock-transfectants (Puro) and DNase $\gamma$-antisense cells (DNase $\gamma$-AS) were cultured in DM containing $50 \mathrm{ng} / \mathrm{ml} \mathrm{NGF}$ for 6 days. The resulting cultures were shifted to DM containing $100 \mathrm{ng} / \mathrm{ml}$ anti-NGF antibody, harvested at the indicated times after NGF withdrawal, and subjected to DNA fragmentation analysis (upper panels) and Western blot analysis for PARP-1 (lower panels) as described under Materials and Methods. Closed and open arrowheads indicate intact $(116 \mathrm{kDa})$ and cleaved $(85 \mathrm{kDa})$ forms of PARP-1, respectively

frequently used cells used to study the function of PNS neurons; ${ }^{28}$ however, little is known about the features of their cell death. We found that a large fraction of N1E-115 cells die by typical apoptosis during the early phase of neuronal differentiation. In addition, cells that escape cell death acquire an apoptosis-resistant phenotype and survive for an extended period in culture. On the basis of these results, N1E-115 cells are proposed to be an ideal model system in which to study the control of cell death and survival during neuronal differentiation.

The spatial and temporal distribution of apoptotic neurons were determined previously in developing murine cerebral cortex. ${ }^{29}$ As expected, many dying cells were detected in the postmitotic layers in which initial synaptic connections are being formed. Interestingly, many more apoptotic cells were

a

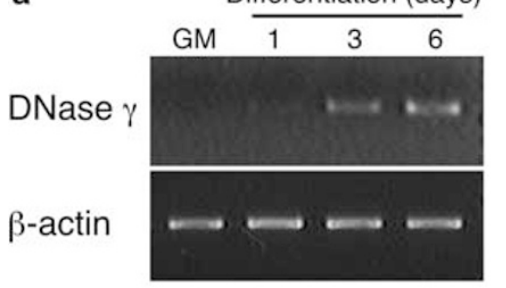

C
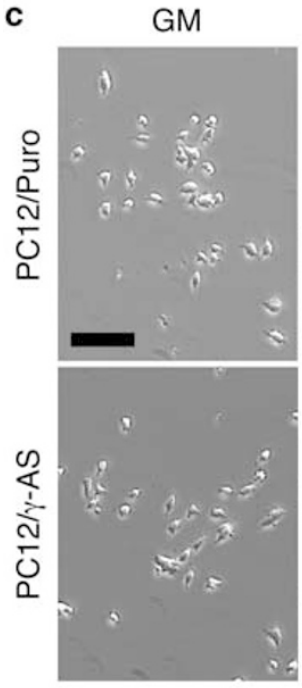

DM (Day 6)
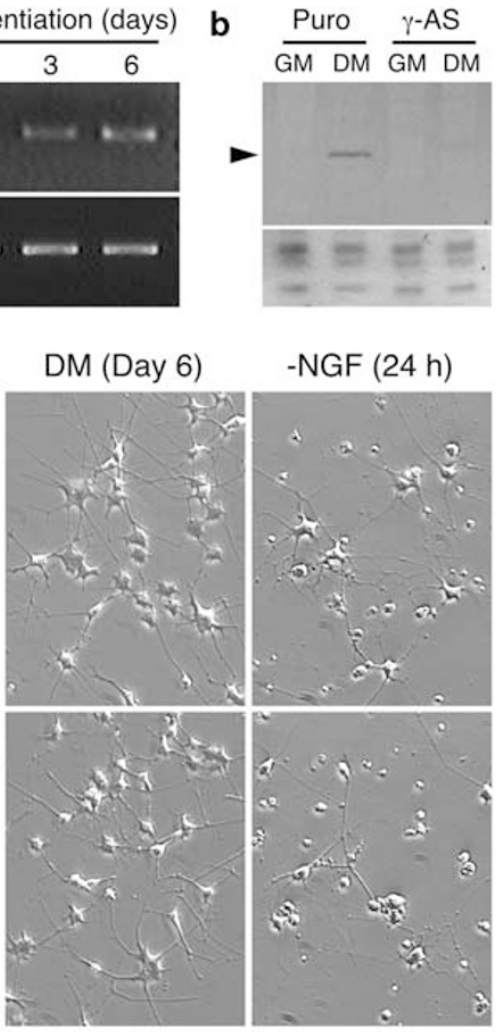

d

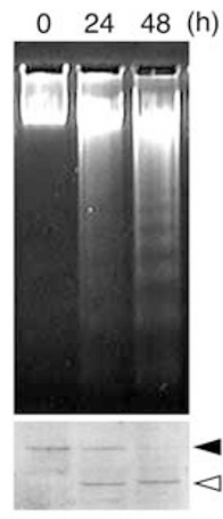

Puro

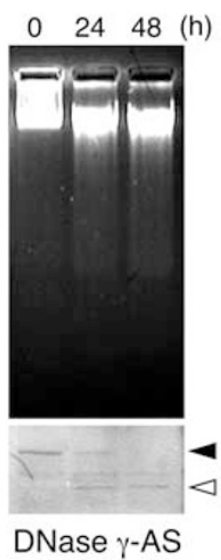


found within a proliferative region, the cortical ventricular zone. Consistent with this observation, neuronal apoptosis was also detected in proliferative regions of the retina and spinal cord. ${ }^{30,31}$ The physiological importance of apoptosis in such naive neurons is currently unknown; however, these observations indicate that a large fraction of proliferating neuroblasts and/or early differentiating neurons are eliminated by apoptosis during the development of both CNS and PNS. These observations suggest the possible involvement of DNase $\gamma$ in apoptosis of CNS neurons. In fact, an induction of DNase $\gamma$ is observed in CNS-derived neuroblastoma B50 cells when the cells are shifted to differentiating conditions. Furthermore, DNase $\gamma$ expression is transiently induced in developing brain during the period of neurogenesis and naturally occurring cell death (our unpublished observations).

In a previous study, DNase $\gamma$ was shown to produce DNA ladders in apoptosis associated with the myogenic differentiation of C2C12 cells. ${ }^{26}$ Therefore, the control of apoptotic DNA fragmentation by DNase $\gamma$ is found to be conserved between muscle and neuronal cell types in the differentiation state. In adult mice, DNase $\gamma$ expression is quite low in skeletal muscle and brain, suggesting that little DNase $\gamma$ exists in syncitial muscle fibers or mature networking neurons. That is, the occurrence of DNase $\gamma$-dependent apoptosis is likely to be limited to specific stages of myogenesis and neurogenesis.

The differentiation-dependent contributions of CAD and DNase $\gamma$ are also an important finding of this study. A high expression of CAD is detected in proliferating N1E-115 cells, and apoptotic DNA fragmentation induced by STS under proliferating conditions is abolished by the expression of a caspase-resistant ICAD mutant. In contrast, DNase $\gamma$ is induced during neuronal differentiation and its downregulation by antisense RNA results in the supression of DNA fragmentation both in spontaneous apoptosis during differentiation and in STS-induced apoptosis in neuronally differentiated N1E-115 cells. Thus, apoptotic DNA fragmentation in N1E-115 cells is found to be catalyzed by CAD and DNase $\gamma$ under proliferating and differentiating conditions, respectively. It has been shown that the expression level of ICAD/DFF45 mRNA remains almost constant, whereas that of CAD/DFF40 varies among tissues and cell types. ${ }^{32-34}$ Interestingly, a previous study revealed that the mRNA for CAD is detected at high levels in fetal and newborn cerebellum, but at low levels in adult cerebellum in rat. ${ }^{35}$ The downregulation of CAD expression during neuronal differentiation may account for its expression profile in developing cerebellum.

To date, the regulation of apoptotic DNA fragmentation has been well explained by the CAD/ICAD system in many apoptotic cases; however, evidence supporting the involvement of other DNases is also accumulating. A mitochondrial DNase, endonuclease $G$, has been shown to catalyze nucleosomal DNA fragmentation in a caspase-independent apoptotic pathway initiating from mitochondria. ${ }^{12}$ Furthermore, a recent study revealed that a tumor metastasis suppressor, NM23-H1, is a granzyme A-activated DNase, and a nucleosome assembly protein, SET, is its specific inhibitor. ${ }^{36} \mathrm{NM} 23-\mathrm{H} 1$ is activated by the granzyme Amediated cleavage of SET, and catalyzes single-strand nicking of chromosomal DNA during cytotoxic T lymphocytemediated apoptosis. Although the activation mechanism of
DNase $\gamma$ remains unclear, its apoptotic function is likely to be to produce DNA fragmentation at some specific stages of cellular development. Pathways leading to apoptosis are now revealed to be more complex and multiple than once predicted. Therefore, it may be reasonable that multiple DNases are selectively used depending on the upstream apoptotic pathways.

In this study, we have revealed the involvement of DNase $\gamma$ in neuronal differentiation and NGF-deprivation-induced apoptosis in N1E-115 and PC12 cells, respectively. Furthermore, our results indicate that apoptotic DNA fragmentation in N1E-115 cells is catalyzed by either CAD or DNase $\gamma$ depending on the differentiation state. The reason for the selective usages of CAD and DNase $\gamma$ in neuronal apoptosis is currently unknown. In addition, it remains elusive whether this is widely conserved among neuronal cell types or is a specific feature of some limited lineage. More studies are required to provide answers to the above questions; however, such information provides important clues for understanding the control of apoptosis in developing neurons.

\section{Materials and Methods}

\section{Cell culture}

Mouse neuroblastoma N1E-115 cells were maintained in growth medium (GM, Dulbecco's modified Eagle's medium (DMEM) supplemented with $10 \%$ fetal calf serum (FCS)). To induce neuronal differentiation, $1 \times 10^{5}$ cells grown in $60 \mathrm{~mm}$ poly-L-lysine-coated dish were shifted to differentiation medium (DM, DMEM alone) and cultured for the indicated number of days with daily medium replacement. To induce apoptosis by STS treatment, cells were washed twice with fresh medium and cultured for the indicated time in the presence of $0.5 \mu \mathrm{M}$ STS.

Rat pheochromocytoma PC12 cells were maintained in GM (DMEM supplemented with 5\% FCS and 10\% horse serum). Neuronal differentiation was induced by shifting the culture, $5 \times 10^{4}$ cells grown in $60 \mathrm{~mm}$ poly-L-lysine-coated dish, to DM (DMEM containing $0.1 \mathrm{mM}$ dibutyryl-cyclic AMP) supplemented with $50 \mathrm{ng} / \mathrm{ml}$ of the $2.5 \mathrm{~S}$ form of mouse NGF (Promega) and cultured for the indicated number of days. The medium of the differentiation cultures was replaced with fresh medium every other day. In the case of the induction of apoptosis, neuronally differentiated PC12 cells were washed twice, placed in fresh DM, and cultured for the indicated time in the presence of $100 \mathrm{ng} / \mathrm{ml}$ anti-NGF mAb (Chemicon). The morphologies of PC12 cells were observed under a phase-contrast microscope (Olympus, CK40).

\section{Indirect immunofluorescence}

DNase $\gamma$ and NF-H were detected in situ using hg302 anti-DNase $\gamma \mathrm{mAb}$ $(2 \mu \mathrm{g} / \mathrm{ml}$, Oncogene) and anti-NF-H mAb (1:1000 dilution, Sigma), respectively, as described previously with some modifications. ${ }^{26}$ In brief, cells grown on sterile poly-L-lysine-coated coverslips were fixed with $3.5 \%$ formaldehyde, soaked in ethanol, and, after blocking with DPBS containing $5 \%$ FCS, the resulting cells were incubated with each $\mathrm{mAb}$ at $4^{\circ} \mathrm{C}$ overnight in a humid sealed chamber. After incubation, the cells were washed with DPBS and incubated with FITC-conjugated-anti mouse $\lg \mathrm{G}(1: 300$ dilution, Vector Laboratory) at room temperature for $1 \mathrm{~h}$. After washing with DPBS, the morphology of the cells and fluorescence of FITC were observed under a fluorescence microscope (Olympus, BX60). Digital images were processed with Photoshop 7.0 software (Adobe). 


\section{Apoptosis assays}

Nuclear condensation and the externalization of phosphatidylserine were detected by Hoechst 33342 (Molecular Probes) and FITC-conjugated annexin V (Medical and Biological Laboratories) staining, respectively, as described previously. ${ }^{26}$ To visualize dead cells in a whole culture, trypan blue solution (Sigma) was added to the culture at a final concentration of $0.04 \%$. The cultures were then gently mixed, kept at room temperature for $5 \mathrm{~min}$, and observed under a differential interference contrast (DIC) microscope (Carl Zeizz, Axiovert 135). Apoptotic DNA fragmentation was analyzed by $1.8 \%$ agarose gel electrophoresis as described previously. ${ }^{26}$ Relative cell number and relative viable cells were estimated by counting the cells attached to the culture dishes at the indicated times. Values are the averages of three independent experiments and are relative to the initial cell number in each experiment.

\section{Western blot}

Western analyses were performed using anti-PARP (1:1000 dilution, Wako), anti ICAD (1: 1000 dilution, Medical and Biological Laboratories), anti-CAD (1: 1000 dilution, Millennium Biotechnology), anti-actin (1:500 dilution, Santa Cruz Biotechnology) polyclonal antibodies as described previously. ${ }^{26}$ In case of CAD and $\beta$-actin detections, blots were visualized using ECL Plus Western blotting detection reagents (Amersham pharmacia) according to the manufacturer's protocol.

\section{RT-PCR analysis}

RT-PCR analysis was performed using total RNA extracted from the indicated cells as described previously. ${ }^{26}$ PCR amplification was carried out for the indicated number of cycles, each cycle consisting of denaturation at $94^{\circ} \mathrm{C}$ for $30 \mathrm{~s}$, annealing at $62^{\circ} \mathrm{C}$ for $30 \mathrm{~s}$, and extension at $72^{\circ} \mathrm{C}$ for $1 \mathrm{~min}$. The primers used were: CAD (34 cycles), $5^{\prime}$. CAGGACCCATCCTGGTTTGAAGGT-3' (sense) and 5' -GATCCTGTCTGAGGCCGATAGATC-3' (antisense); ICAD (30 cycles), 5'-GGCCATTGATAAGTCCCTGAC-3' (sense) and 5'-CCTGAGTGAATGCAAGCTCTG-3' (antisense); DNase $\gamma$ (32 cycles), 5'-CACGTACAAAGAGCAGTATGC-3' (sense) and 5'-CGAATGTTCTGCCAGGCCTTC-3' (antisense); and $\beta$-actin (25 cycles), $5^{\prime}$-CACCTTCTACAATGAGCTGCG- $3^{\prime}$ (sense) and 5'-CTTGCTGATCCACATCTGCTG-3' (antisense).

\section{Assay of DNase $\gamma$ activity}

DNase $\gamma$ activity was analyzed by activity gel assay as described previously. ${ }^{26}$ The in-gel reaction was performed in reaction buffer $(10 \mathrm{mM}$ Tris- $\mathrm{HCl}$ (pH 7.8), $1 \mathrm{mM}$ 2-mercaptoethanol, $3 \mathrm{mM} \mathrm{CaCl}, 3 \mathrm{mM} \mathrm{MgCl}$ ) overnight at $50^{\circ} \mathrm{C}$. After the observation of DNase activity, the resulting gel was subjected to Coomassie Brilliant Blue (CBB) staining to confirm equal lane loading.

\section{Establishment of N1E-115/ICAD-CR cells}

A cDNA fragment containing the entire open reading frame of mouse ICAD was generated by PCR and subcloned into pUC19 vector. PCR primers used were: 5'-GTCGACCACCATGGAGCTGTCGCGG-3' (sense) and 5'GTCGACACGAGGAGTCTCGTTTGG-3' (antisense). Sall sites flanking the coding sequence are shown in bold letters. Two caspase-cleavage sites, the 117th and 224th aspartic acid residues, were converted to glutamic acid by site-directed mutagenesis using an LA PCR in vitro Mutagenesis kit (Takara). Mutation oligonucleotides used were: D117E,
5'-ATGAGCCGGAGAGCAGGGCA-3'; and D224E, 5'-ATGCAGTTGAGACAGGCGTC-3'. Mutated bases are underlined. The resulting insert was excised by Sall digestion and recloned into the Xhol site of pcDNA 3.1 (C) vector to fuse an extra sequence for a C-terminal Myc and His $\times 6$ tags. The open reading flame for ICAD-CR-Myc-His was amplified by PCR from the vector and recloned into $\mathrm{pCAG} /$ puro plasmid ${ }^{26}$ to generate an expression vector for a caspase-resistant form of ICAD (pCAG/ICAD-CRMyc-His). The PCR primers used were: 5'-GTCGACCACCATGGAGCTGTCGCGG-3' (sense) and 5'-GTCGACTAGAAGGCACAGTCGAGGCTG-3' (antisense). Sall sites flanking the coding sequence are shown in bold letters.

Proliferating N1E-115 cells were transfected with pCAG/ICAD-CR-MycHis using FuGENE6 transfection reagent (Roche) according to the manufacturer's protocol, and cells stably integrated by the transgene were selected by puromycin resistance $(3 \mu \mathrm{g} / \mathrm{ml})$. The resulting cells were cloned by two cycles of colony isolation, and several independent clones were obtained. Subcultures of the clones were subjected to Western blot using anti-ICAD pAb and cells expressing high levels of the ICAD-CR protein were selected. As a consequence, we obtained N1E-115/ICAD-CR cells (clone 1.10). A mock transfectant, N1E-115/puro cells, was obtained by the same method, except for selection by ICAD-CR expression, and used in control experiments.

\section{Antisense RNA-mediated suppression of DNase $\gamma$}

Proliferating N1E- 115 cells were transfected with pCAG-mDNase $\gamma$ AS, an antisense RNA expression vector for DNase $\gamma,{ }^{26}$ selected by puromycin resistance $(3 \mu \mathrm{g} / \mathrm{ml})$, to obtain several independent clones of DNase $\gamma$ antisense cells as described above. Subcultures of the clones were allowed to differentiate in DM for 4 days, and clones whose DNase $\gamma$ activity was efficiently suppressed were selected. DNase $\gamma$ activity was analyzed by activity gel assay as described above. As a result, we established several DNase $\gamma$-knockdown cells (N1E-115/ $\gamma$-AS), and clone 1.15 was subjected to further analyses.

A cDNA fragment containing the entire open reading frame of rat DNase $\gamma$ was excised from prDNase $\gamma$-Myc-His vector ${ }^{27}$ by Xhol digestion. The fragment was then subcloned into $\mathrm{pCAG/puro} \mathrm{in} \mathrm{the} \mathrm{reverse}$ orientation and an antisense RNA expression vector for rat DNase $\gamma$ (pCAG-rDNase $\gamma A S$ ) was obtained. Proliferating PC12 cells were transfected with pCAG-rDNase $\gamma$ AS, and PC12/ $\gamma$-AS cells (clone 3.08) were established as described above. PC12 cells stably integrated by the transgene were selected by puromycin resistance $(2 \mu \mathrm{g} / \mathrm{ml})$. A mock transfectant, PC12/puro cells, was obtained by transfection with empty vector and used in control experiments.

\section{Acknowledgements}

We thank Dr. Junji Yamauchi for helpful discussions, and Miss Mana Kawabata and Miss Aya Maehara for their excellent technical assistance. This work was funded in part by a Grant-in-Aid for Scientific Research from the Ministry of Education, Culture, Sports, Science, and Technology of Japan.

\section{References}

1. Davies AM (1994) The role of neurotrophins in the developing nervous system. J. Neurobiol. 25: 1334-1348 
2. Oppenheim RW (1989) The neurotrophic theory and naturally occurring motoneuron death. Trends. Neurosci. 12: 252-255

3. Voyvodic JT (1996) Cell death in cortical development: how much? Why? So what? Neuron 16: 693-696

4. Ellis RE, Jacobson DM and Horvitz HR (1991) Mechanisms and functions of cell death. Annu. Rev. Cell. Biol. 7: 663-698

5. Meier P, Finch A and Evan G (2000) Apoptosis in development. Nature 407: 796-801

6. Kerr JFR, Wyllie AH and Currie AR (1972) Apoptosis: a basic biological phenomenon with wide-ranging implications in tissue kinetics. Br. J. Cancer 26 : 239-257

7. Budihardjo I, Oliver H, Lutter M, Luo X and Wang X (1999) Biochemical pathways of caspase activation during apoptosis. Annu. Rev. Cell Dev. Biol. 15: 269-290

8. Schlegel RA and Williamson P (2001) Phosphatidylserine, a death knell. Cell Death Differ. 8: 551-563

9. Zhang JH and Xu M (2000) DNA fragmentation in apoptosis. Cell Res. 10: 205211

10. Kaufmann SH, Mesner Jr PW, Samejima K, Tone S and Earnshaw WC (2000) Detection of DNA cleavage in apoptotic cells. Methods Enzymol. 22: 3-15

11. Enari M, Sakahira H, Yokoyama H, Okawa K, Iwamatsu A and Nagata S (1998) A caspase-activated DNase that degrades DNA during apoptosis, and its inhibitor ICAD. Nature 391: 43-50

12. Li LY, Luo $X$ and Wang $X$ (2001) Endonuclease $G$ is an apoptotic DNase when released from mitochondria. Nature 412: 95-99

13. Liu X, Zou H, Slaughter $C$ and Wang X (1997) DFF, a heterodimeric protein that functions downstream of caspase-3 to trigger DNA fragmentation during apoptosis. Cell 89: 175-184

14. Rauch F, Polzar B, Stephan H, Zanotti S, Paddenberg R and Mannherz HG (1997) Androgen ablation leads to an upregulation and intranuclear accumulation of deoxyribonuclease I in rat prostate epithelial cells paralleling their apoptotic elimination. J. Cell Biol. 137: 909-923

15. Rodriguez AM, Rodin D, Nomura H, Morton CC, Weremowicz $S$ and Schneider MC (1997) Identification, localization, and expression of two novel human genes similar to deoxyribonuclease I. Genomics 42: 507-513

16. Shiokawa $D$ and Tanuma $S$ (1998) Molecular cloning and expression of a cDNA encoding an apoptotic endonuclease DNase $\gamma$. Biochem. J. 332: 713-720

17. Mcllroy D, Tanaka M, Sakahira H, Fukuyama H, Suzuki M, Yamamura K, Ohsawa Y, Uchiyama Y and Nagata S (2000) An auxiliary mode of apoptotic DNA fragmentation provided by phagocytes. Genes Dev. 14: 549-558

18. Kawane K, Fukuyama H, Yoshida H, Nagase H, Ohsawa $\mathrm{Y}$, Uchiyama $\mathrm{Y}$, Okada K, lida T and Nagata S (2003) Impaired thymic development in mouse embryos deficient in apoptotic DNA degradation. Nat. Immunol. 4: 138-144

19. Sakahira H, Enari M and Nagata S (1998) Cleavage of CAD inhibitor in CAD activation and DNA degradation during apoptosis. Nature 391: 96-99

20. Zhang J, Liu X, Scherer DC and van Kaer L (1998) Resistance to DNA fragmentation and chromatin condensation in mice lacking the DNA fragmentation factor 45. Proc. Natl. Acad. Sci. USA 95: 12480-12485

21. Cohen JJ and Duke RC (1984) Glucocorticoid activation of a calciumdependent endonuclease in thymocyte nuclei leads to cell death. J. Immunol. 132: $38-42$
22. Nikonova LV, Nelipovich PA and Umansky SR (1982) The involvement of nuclear nucleases in rat thymocyte DNA degradation after gamma-irradiation. Biochim. Biophys. Acta 699: 281-289

23. Shiokawa D, Ohyama H, Yamada T, Takahashi K and Tanuma S (1994) Identification of an endonuclease responsible for apoptosis in rat thymocytes. Eur. J. Biochem. 226: 23-30

24. Shiokawa D and Tanuma S (2001) Characterization of human DNase I family endonucleases and activation of DNase $\gamma$ during apoptosis. Biochemistry 40: $143-152$

25. Yakovlev AG, Wang G, Stoica BA, Simbulan-Rosenthal CM, Yoshihara $K$ and Smulson ME (1999) Role of DNAS1L3 in $\mathrm{Ca}^{2+}$ - and $\mathrm{Mg}^{2+}$-dependent cleavage of DNA into oligonucleosomal and high molecular mass fragments. Nucleic Acids Res. 27: 1999-2005

26. Shiokawa D, Kobayashi T and Tanuma S (2002) Involvement of DNase $\gamma$ in apoptosis associated with myogenic differentiation of $\mathrm{C} 2 \mathrm{C} 12$ cells. J. Biol. Chem. 277: 31031-31037

27. Shiokawa D, Tanaka M, Kimura T, Hashizume K, Takasawa R, Ohyama $H$, Fujita K, Yamada T and Tanuma S (2000) Characterization of two DNase $\gamma$ specific monoclonal antibodies and the in situ detection of DNase $\gamma$ in the nuclei of apoptotic rat thymocytes. Biochem. Biophys. Res. Commun. 275: 343-349

28. Amano T, Richelson E and Nirenberg M (1972) Neurotransmitter synthesis by neuroblastoma clones (neuroblast differentiation-cell culture-choline acetyltransferase-acetylcholinesterase-tyrosine hydroxylase-axons-dendrites). Proc. Natl. Acad. Sci. USA 69: 258-263

29. Blaschke AJ, Staley K and Chun J (1996) Widespread programmed cell death in proliferative and postmitotic regions of the fetal cerebral cortex. Development 122: $1165-1174$

30. Homma S, Yaginuma H and Oppenheim RW (1994) Programmed cell death during the earliest stages of spinal cord development in the chick embryo: a possible means of early phenotypic selection. J. Comp. Neurol. 345: 377-395

31. Young RW (1984) Cell death during differentiation of the retina in the mouse. J. Comp. Neurol. 229: 362-373

32. Luciano F, Ricci JE, Herrant M, Bertolotto $C$, Mari B, Cousin JL and Auberger $P$ (2002) $T$ and $B$ leukemic cell lines exhibit different requirements for cell death: correlation between caspase activation, DFF40/DFF45 expression, DNA fragmentation and apoptosis in T cell lines but not in Burkitt's lymphoma. Leukemia 16: 700-707

33. Mukae N, Enari M, Sakahira H, Fukuda Y, Inazawa J, Toh $\mathrm{H}$ and Nagata S (1998) Molecular cloning and characterization of human caspase-activated DNase. Proc. Natl. Acad. Sci. USA 95: 9123-9128

34. Zhang J, Wang X, Bove KE and Xu M (1999) DNA fragmentation factor 45deficient cells are more resistant to apoptosis and exhibit different dying morphology than wild-type control cells. J. Biol. Chem. 274: 37450-37454

35. Cao G, Pei W, Lan J, Stetler RA, Luo Y, Nagayama T, Graham SH, Yin XM, Simon RP and Chen J (2001) Caspase-activated DNase/DNA fragmentation factor 40 mediates apoptotic DNA fragmentation in transient cerebral ischemia and in neuronal cultures. J. Neurosci. 21: 4678-4690

36. Fan Z, Beresford PJ, Oh DY, Zhang D and Lieberman J (2003) Tumor suppressor NM23-H1 is a granzyme A-activated DNase during CTL-mediated apoptosis, and the nucleosome assembly protein SET is its inhibitor. Cell 112: $659-672$ 